\title{
Hybrid achromatic spectacle lens
}

\author{
Marek ZaJAC
}

Institute of Physics, Technical University of Wrocław, Wybrzeże Wyspiańskiego 27, PL 50-370 Wrocław, Poland, e-mail: marek.zajac@pwr.wroc.pl.

\begin{abstract}
Spectacle lens is a very particular optical imaging element of great practical importance. Although its construction is very simple, some demands are specific (in particular, remarkable shift of an output pupil). A number of classic spectacle lens designs have been known for a long time, however some new possibilities of aberration correction appear if we use a hybrid (diffractive-refractive). Hybrid lens is an optical system composed of a classic refractive ("glass") lens and a diffractive microstructure deposited on one of its surfaces. Imaging properties of such lens can be expressed in terms of dimensionless parameters: $\zeta=-\rho_{1} / \rho_{2}$ (describing the shape of a refractive part), $\beta=z_{\alpha} / z_{\beta}$ (describing the geometry of diffraction fringes) and $\eta=\Phi_{D} / \Phi_{R}$ (describing the distribution of focusing power between a diffractive and refractive part). By a proper choice of the parameter $\eta$ we can compensate chromatic aberration. Thanks to the other free parameters astigmatism can also be corrected. In this contribution the possibilities of particular Seidel aberration correction of a hybrid lens are presented. As an illustration some examples of spectacle hybrid lenses are shown and their imaging characteristics are compared with imaging characteristics of commercially available refractive lenses.
\end{abstract}

Keywords: hybrid lens, spectacle lens, aberrations.

\section{Introduction}

Single lens of spherical surfaces has limited possibilities of aberration correction. In particular, it is impossible to correct chromatic aberration. This effect is very inconvenient for spectacle wearers [1]-[3]. In conventional optics it is possible to correct chromatic aberration, but at least two lenses are necessary, if considering thin lenses only. Unfortunately, doublets are not useful for spectacles. Correction of chromatic aberration can be easily obtained if using a hybrid lens [4]-[9].

As a hybrid lens, in this paper, a refractive lens made of glass of the refractive index $n$ and having spherical surfaces of curvature radii equal to $\rho_{1}$ and $\rho_{2}$ is considered. On one of its surfaces (let us assume: the first) there is deposited a diffractive microstructure of fringe geometry equivalent to the interference field generated by two spherical waves of the wavelength $\lambda$ originated from the points $P_{\alpha}$ and $P_{\beta}$, located on the lens axis in the distances $z_{\alpha}$ and $z_{\beta}$ from the lens (see Fig. 1). This structure can be manufactured interferometrically or, better, synthetically with a 


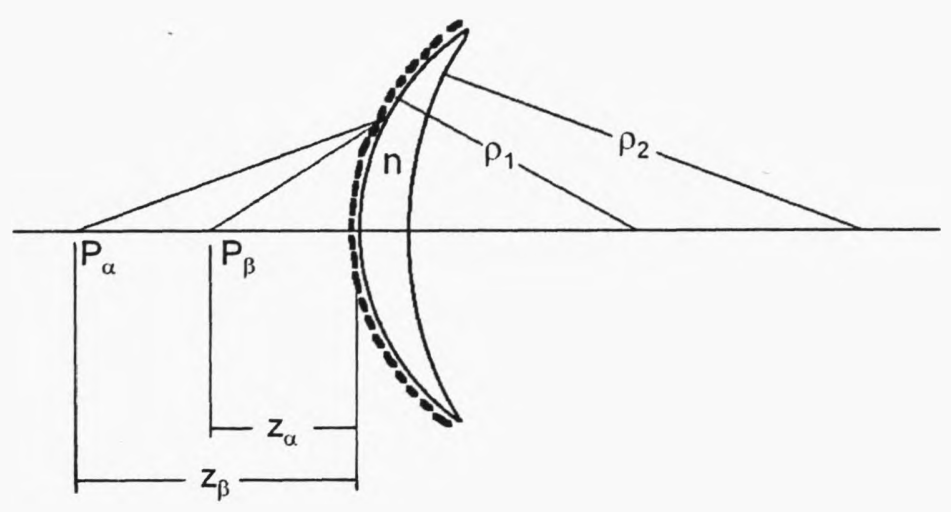

Fig. 1. Hybrid lens.

kinoform profile assuring high diffraction efficiency. Object point to be imaged is located in the point $P$ of coordinates $(y, s)$ and its image is formed in the point $P^{\prime}$ of coordinates $\left(y^{\prime}, s^{\prime}\right)$.

\section{Monochromatic aberrations of hybrid lens}

In the following text we use dimensionless parameters describing construction parameters of the lens, as well as imaging geometry. The shape of a refractive part is defined by the ratio of curvature radii of both surfaces $\zeta=-\rho_{1} / \rho_{2}$. We remind that $\zeta<1$ describes a positive meniscus lens, and $1<\zeta<0$ describes a negative meniscus lens. The diffractive structure is defined by the parameter $\beta=z_{\alpha} / z_{\beta}$, and

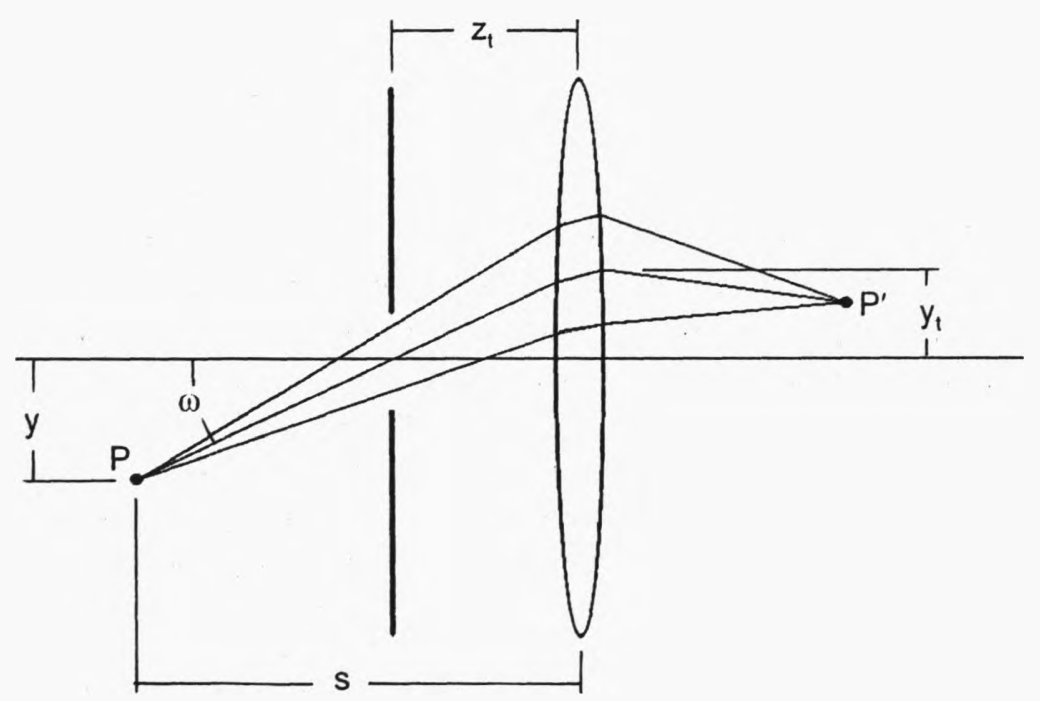

Fig. 2. Imaging by a lens with shifted input pupil. 
the ratio of diffraction-refraction focusing powers is given by the parameter $\eta=\Phi_{D} / \Phi_{R}$. The location of an object point is defined by the parameters expressing the field-of-view angle $\omega=y / s$ and normalized object distance $v=\left(1 / \Phi_{s}\right)$.

It can be shown [5], [6], [9]-[13] that III-order Seidel aberration coefficients ( $S$ for spherical aberration, $C$ for coma, $F$ for field curvature and $A$ for astigmatism) of the hybrid lens with the diffractive microstructure deposited on the first surface and an input pupil in contact with this surface are expressed as follows:

$$
\begin{aligned}
& S=\Phi^{3}\left\{v\left(v-\frac{1}{(n-1)(1+\zeta)(1+\eta)}\right)^{2}\right. \\
& -(v+1)\left(v+1+\frac{\zeta}{(n-1)(1+\zeta)(1+\eta)}\right)^{2} \\
& -\frac{1}{n^{2}}\left(v+\frac{1}{(1+\zeta)(1+\eta)}+\frac{\eta}{1+\eta}\right)\left(v+\frac{\eta}{1+\eta}-\frac{1}{(n-1)(1+\zeta)(1+\eta)}\right)^{2} \\
& +\frac{1}{n^{2}}\left(v+\frac{1}{(1+\zeta)(1+\eta)}+\frac{\eta}{1+\eta}\right)\left(v+\frac{\eta}{1+\eta}-\frac{n \zeta+n-1}{(n-1)(1+\zeta)(1+\eta)}\right)^{2} \\
& +\frac{\eta}{(1+\eta)^{3}(1-\beta)}\left[\left(\frac{\eta}{(1-\beta)}-\frac{1}{(n-1)(1+\zeta)}\right)^{2}\right. \\
& \left.\left.-\beta\left(\frac{\beta \eta}{(1-\beta)}-\frac{1}{(n-1)(1+\zeta)}\right)^{2}\right]\right\} \text {, } \\
& C=\Phi^{2} \omega\left[v\left(v-\frac{1}{(n-1)(1+\zeta)(1+\eta)}\right)\right. \\
& -(v+1)\left(v+1+\frac{\zeta}{(n-1)(1+\zeta)(1+\eta)}\right) \\
& \left.+\frac{1}{n(n-1)(1+\eta)}\left(v+\frac{1}{(1+\zeta)(1+\eta)}+\frac{\eta}{1+\eta}\right)\right] \text {, } \\
& F=-\Phi \omega^{2}\left[1+\frac{1}{n(\eta+1)}\right] \\
& A=-\Phi \omega^{2} .
\end{aligned}
$$

The coefficient describing distortion equals zero by identity. 
The above expressions were derived under assumption that the object pupil coincides with the lens surface. Otherwise the input pupil shift on the distance $z_{t}$ in front of the lens results in the perpendicular shift of the center of the lens acting area of the value $y_{t}$ (see Fig. 2)

$$
y_{i}=\frac{\omega z_{t}}{1-v z_{t}}
$$

This causes a change in aberration coefficients. Resulting aberration coefficients for the lens with a shifted pupil can be expressed by the respective coefficients of the lens with a pupil in contact and value $y_{t}$ :

$$
\begin{aligned}
& S_{t}=\bar{S}, \\
& C_{t}=\bar{C}-y_{t} \bar{S}, \\
& A_{t}=\bar{A}-2 y_{t} \bar{C}+y_{t}^{2} \bar{S}, \\
& F_{t}=\bar{F}-2 y_{t} \bar{C}+y_{t}^{2} \bar{S}, \\
& D_{t}=\bar{D}-2 y_{t} \bar{A}-y_{t} \bar{F}+3 y_{t}^{2} \bar{C}-y_{t}^{3} \bar{S} .
\end{aligned}
$$

Coefficients $\bar{S}, \bar{C}, \bar{A}, \bar{F}, \bar{D}$ are formally identical to the coefficients $S, C, A, F, D$ but all coordinates $y$ are replaced by $y-y_{t}$ therein.

In the case of spherical aberration the respective expression does not depend on the coordinate $y$, so $\bar{S} \equiv S$. Similarly for the rest of coefficients if the object is located in infinity. The value $y$ appears explicitly in the above equations only for finite object distance since $\omega=y / s$.

In consequence the III-order aberration coefficients of a hybrid lens can be expressed by a number of parameters depending on the construction of the lens $(\eta, \zeta, \beta)$ as well as imaging geometry $\left(v, z_{t}\right)$. Proper choice of these parameters allows to correct chosen aberrations.

\section{Correction of chromatic aberration}

The main advantage of a hybrid lens is in the possibility of chromatic aberration correction. The condition for achromatisation for two wavelengths $\lambda_{1}$ and $\lambda_{2}$ is [6]

$$
\eta_{0}=\left.\frac{\Phi_{D}}{\Phi_{R}}\right|_{\lambda_{0}}=\frac{-v_{D}}{v_{R}},
$$


where $v_{R}$ is Abbe number of the lens material and $v_{D}$ its equivalent for diffractive structure defined as

$$
v_{D}=\frac{\lambda_{0}}{\lambda_{1}-\lambda_{2}}
$$

where $\lambda_{0}$ is the base wavelength.

The Abbe number for diffractive structure has an opposite sign than the Abbe number for optical glass. In consequence, both parts of an achromatic hybrid doublet have the same sign of focusing power. Moreover, since the value of the Abbe number of a diffractive part is smaller than this for a refractive part (approximately on an order of magnitude), the diffractive structure bears only small part of the overall focusing power, which is advantageous for technological reasons.

Unfortunately the hybrid lens has a considerable secondary spectrum.

$$
\frac{\Delta s^{\prime}}{s^{\prime 2}}=\frac{P_{R}-P_{D}}{v_{R}-v_{D}} \Phi=\gamma \Phi
$$

where $s^{\prime}$ is an image distance, $P_{R}$ and $P_{D}$ are partial dispersions of glass and its refractive structure equivalent

$$
P_{D}=\frac{\lambda_{1}-\lambda_{0}}{\lambda_{1}-\lambda_{2}}
$$

\section{Correction of monochromatic aberration}

The imaging properties of an achromatic hybrid lens are described by three parameters $\eta_{0}, \zeta$ and $\beta$; while the last two are still unspecified. Therefore we can compensate two monochromatic aberrations. In the case of a spectacle lens it is reasonable to correct astigmatism. From the Eq. (7) it is seen that it is easy to find condition for astigmatism correction if additionally $S=0$.

Inserting Eqs. (1), (2) and (5) into Eq. (7) and assuming that $S=0$ we receive the equation from which the value of the parameter $\zeta_{0}$ assuring correction of astigmatism can be found

$$
\begin{aligned}
1 & =\frac{z_{t} \Phi}{1-v z_{t}}\left[v\left(v-\frac{1}{(n-1)(1+\zeta)\left(1+\eta_{0}\right)}\right)\right. \\
& -(v+1)\left(v+1+\frac{\zeta}{(n-1)(1+\zeta)\left(1+\eta_{0}\right)}\right) \\
& \left.+\frac{1}{n(n-1)\left(1+\eta_{0}\right)}\left(v+\frac{1}{(1+\zeta)\left(1+\eta_{0}\right)}+\frac{\eta_{0}}{1+\eta_{0}}\right)\right]
\end{aligned}
$$


For the object in infinity $(v=0)$ we have the following solution

$$
\zeta_{0}=\frac{2 z_{t} \Phi\left[n\left(1+\eta_{0}\right)+1\right]}{n(n-1)\left(1+\eta_{0}\right)^{2}+\left[(n-1)\left(1+\eta_{0}\right)+1\right] 2 z_{t} \Phi} .
$$

For near vision, i.e., if $v \neq 0$, the respective formula becomes rather complicated and hardly readable, so we do not give it here. However there are no problems in its exploiting if using any computer program for symbolic calculations.

Finally we have to find the value of the parameter $\beta$, which defines the diffractive microstructure, inserting values $\eta_{0}$ and $\zeta_{0}$ found from Eqs. (11) and (16) into Eq. (1) and solving the equation

$$
S\left(v, n, \zeta_{0}, \eta_{0}, \beta\right)=0 .
$$

Solving this equation by means of any symbolic calculation computer program we get two solutions for $\beta_{0}$. In consequence we have hybrid spectacle lens of corrected sphero-chromatic aberration and astigmatism. Its construction parameters are expressed by the parameters $\eta_{0}, \zeta_{0}, \beta_{0}$ and $\Phi$ as follows:

$$
\begin{aligned}
& \rho_{1}=\left[\frac{\Phi}{n_{0}-1} \frac{1}{\eta_{0}+1} \frac{1}{\zeta_{0}+1}\right]^{-1}, \\
& \rho_{2}=\left[\frac{\Phi}{n_{0}-1} \frac{1}{\eta_{0}+1} \frac{\zeta_{0}}{\zeta+1}\right]^{-1}, \\
& z_{\alpha}=\left[\Phi \frac{\eta_{0}}{\eta_{0}+1} \frac{1}{1-\beta_{0}}\right]^{-1}, \\
& z_{\beta}=\left[\Phi \frac{\eta_{0}}{\eta_{0}+1} \frac{\beta_{0}}{1-\beta_{0}}\right]^{-1} .
\end{aligned}
$$

\section{Examples}

In order to illustrate the above calculations we analyzed some examples of positive lens of high focusing power $(\Phi=+10 \mathrm{D})$ made of two kinds of optical glass: standard crown type glass and high index glass. In spite of remarkable chromatic aberration optical glasses of $n>1.7$ are now widely used since they allow to design thinner lenses of more flat surfaces.

The construction parameters of thin achromatic hybrid lenses of corrected spherical aberration and astigmatism are calculated according to the procedure presented in previous paragraph. As a base wavelength $\lambda_{e}=0.5460 \mathrm{~nm}$ has been taken, and the achromatisation interval is limited by $\lambda_{F}=0.4861 \mathrm{~nm}$ and $\lambda_{d}=0.5876 \mathrm{~nm}$. Such 



Fig. 3. Refractive lens IZOKRON $15^{\oplus}$ and its imaging characteristics $\left(\Phi=10 \mathrm{D}, z_{t}=25.0 \mathrm{~mm}\right.$,





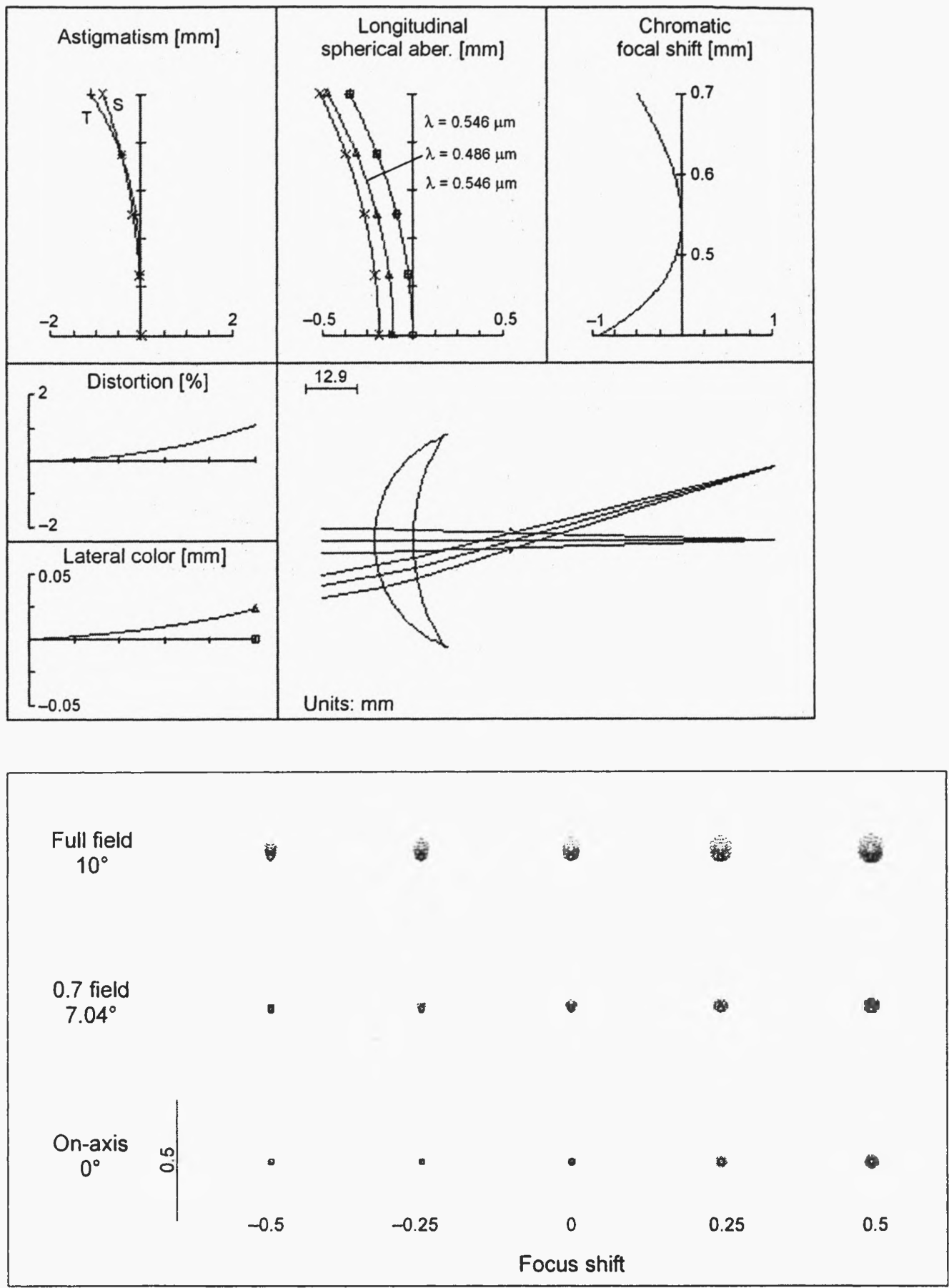

Fig. 4. Hybrid lens IZOKRON $15^{\circledR}$ (crown glass) and its imaging characteristics ( $\Phi=10 \mathrm{D}, z_{\mathrm{t}}=25.0 \mathrm{~mm}$, $\omega=10^{\circ}, n=1.5252, \rho_{\mathrm{l}}=28.9 \mathrm{~mm}, \rho_{2}=46.258 \mathrm{~mm}, d=10 \mathrm{~mm}, z_{\alpha}=15.198 \mathrm{~mm}, z_{\beta}=15.328 \mathrm{~mm}$, $S F^{\prime}=100.002 \mathrm{~mm}$ ). 

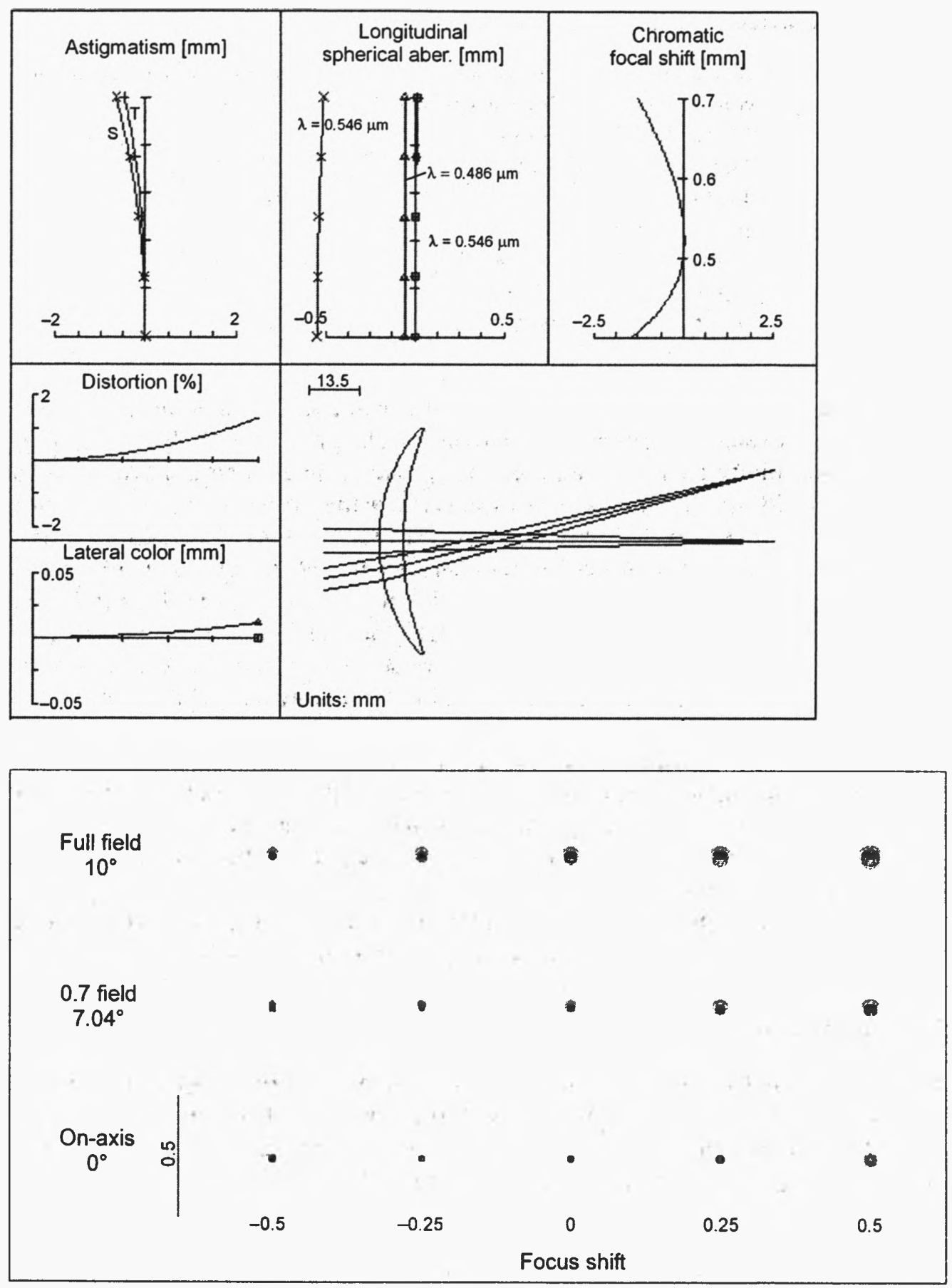

Fig. 5. Hybrid lens IZOKRON $18^{\circledR}$ (high index glass) and its imaging characteristics $(\Phi=10 \mathrm{D}$, $z_{t}=25.0 \mathrm{~mm}, \omega=10^{\circ}, n=1.8002, \rho_{1}=47.0 \mathrm{~mm}, \rho_{2}=86.537 \mathrm{~mm}, d=6.5 \mathrm{~mm}, z_{\alpha}=21.978 \mathrm{~mm}$, $\left.z_{\beta}=22.457 \mathrm{~mm}, S F^{\prime}=99.997 \mathrm{~mm}\right)$. 
$\mathrm{T}$ a b l e. Construction parameters of exemplary lenses.

\begin{tabular}{llll}
\hline Parameter & $\begin{array}{l}\text { Hybrid lens, } \\
\text { crown glass }\end{array}$ & $\begin{array}{l}\text { Hybrid lens, } \\
\text { high index glass }\end{array}$ & $\begin{array}{l}\text { Modified commercial glass lens } \\
\text { IZOKRON 15 }\end{array}$ \\
\hline$n_{d}$ & 1.5252 & 1.8002 & 1.5252 \\
$\rho_{1}$ & 28.9 & 47.0 & 43.75 \\
$\rho_{2}$ & 46.258 & 86.537 & 175.0 \\
$d$ & 10 & 6.5 & 10.2 \\
$z_{\alpha}$ & 15.198 & 21.9780 & - \\
$z_{\beta}$ & 15.328 & 22.457 & - \\
$S F^{\prime}$ & 100.02 & 99.997 & 99.94 \\
\hline
\end{tabular}

choice is justified by the spectral sensitivity of human eye. For photopic (day) vision the eye has maximum sensitivity around the wavelength $\lambda_{e}$. On the blue end of the spectrum sensitivity falls to about $19 \%$ for $\lambda_{F}$ and at the red end to about $27 \%$ for $\lambda_{\mathrm{He}-\mathrm{Ne}}=0.6328 \mathrm{~nm}$ [1]. It has been assumed that the object is placed in infinity (spectacles for distant vision) and astigmatism is corrected for field angle $\omega=10^{\circ}$. Aberration characteristics of the designed lenses were calculated with the help of a typical computer program [14]. Since real lens cannot be infinitely thin the construction parameters have to be changed slightly in order to assure thickness complying with technology demands. New parameters were found in such a way that front focal plane of the lens was unchanged and aberrations remained the same or even smaller. The necessary correction of construction parameters can be easily performed with the help of any lens design program (e.g., [14]).

Typical, commercially available glass lenses were considered for comparison purposes [15]. Due to technology limitations the catalogue lenses not always have optimally corrected aberrations. Therefore we modified slightly their radii of curvature to minimize aberrations.

The construction parameters of exemplary lenses are collected in the Table and the aberration characteristics of these lenses are presented in Figs. 3-5.

\section{Conclusions}

Presented examples prove that it is possible to design a hybrid spectacle lens of relatively high focusing power with astigmatism and chromatic aberration compensation substantially better than in the case of a spherical glass lens. Additionally spherochromatic aberration is corrected.

Acknowledgment - This paper was presented at XIV Polish-Czech-Slovak Optical Conference Wave and Quantum Aspects of Contemporary Optics, Krzyżowa, September 14-17, 2002. 


\section{References}

[1] Kaiser P.M., Boynton R.M., Human Color Vision, OSA, Washington 1996.

[2] Rynders M.C., Navarro R., Losada M.A., Vis. Res. 38 (1998), 513.

[3] Faubert J., Simonet P., Gresset J., Ophthalmic \& Physiolo. Optics 19 (1999), 336.

[4] O'Shea D.C., Appl. Opt. 33 (1994), 8124.

[5] Zajac M., NowaK J., Dubik B., Opt. Appl. 21 (1991), 33.

[6] Котн S., NowaK J., Zajac M., Optik 106 (1997), 63.

[7] Sweatt W.C., J. Opt. Soc. Am. 67 (1977), 803.

[8] Jagoszewskı E., Holographic Optical Elements, Oficyna Wydawnicza Politechniki Wrocławskiej, Wrocław 1995 (in Polish).

[9] Zhao Liping, Lam Yee Loy, Zhou Yan, Yun Zhisheng, Proc. SPIE 3778 (1999), 11.

[10] Nowak J., Pietraszkiewicz K., Zają M., Diffractive Optical Elements, Oficyna Wydawnicza Politechniki Wrocławskiej, Wrocław 1997 (in Polish).

[11] NowaK J, Zają M., Proc. SPIE 3829 (1998), 479.

[12] Zajac M., Sawicz P., NowaK J., Opt. Appl. 31 (2001), 799.

[13] ZaJAC M., Opt. Appl., 31 (2001), 815.

[14] OSLO LIGHT Programme, Sinclair Optics Ltd.

[15] Courtesy JZO Sp.z o.o., Jelenia Gora, Poland.

Received October 29, 2002

in revised form January 30, 2003 